\title{
A design of simple soldering machine
}

\author{
Yinhe Sheng ${ }^{1, a}$, Kun Liu ${ }^{2, b}$, Defu Shao ${ }^{2, c}$ and Xiaoming Feng ${ }^{2, d}$ \\ School of Mechanical Engineering, Jiamusi University, Jiamusi154007, China \\ a1396210151@qq.com, 'blk_liukun@yeah.net, 'sysxk8688@163.com, ${ }^{\mathrm{d}} 1159527506 @ q q . c o m$ \\ Corresponding author: Defu Shao
}

Keywords: PCB, Pin-through-hole (PTH), PIC, PCB file.

\begin{abstract}
A type of soldering machine based on PIC-controlling is introduced in this paper, the high speed micro-controller unit (MCU) with kernel of 16F84A is adopted in PIC. The data is send to lower computer to control three axis motions by computer serial port, the process of automatic soldering is realized. The soldering machine has many advantages such as simple structure and operation, low cost etc.
\end{abstract}

\section{Introduction}

The soldering machine has been widely used in the print circuit board (PCB). Such as wave soldering, re-flow soldering, as well as automatic soldering robot ${ }^{[1]}$. The pin-through-hole (PTH) components are soldered to the PCB when passing through the molten solder. The PCB hole is occupied by molten solder that forms the solder joint, which the pin on the PCB is griped and electrical connection is provided ${ }^{[2]}$. The equipment of soldering has a common feature, such as complex structure, high operation and maintenance costs. Hence, the soldering machine based on PIC MCU-controlling is proposed in the paper, it has the characteristic of low price, high accuracy, and mature technology, which provided a very good idea for traditional soldering machine.

Besides, computer aided design (CAD) has been applied by many researches who obtain excellent performance on mechanical product design ${ }^{[3]}$. CAD, also known as computer-aided design and drafting (CADD), the process of design and design-documentation is performed by the use of computer technology ${ }^{[4]}$. Therefore, the design and structure analysis of the soldering machine is realized by SolidWorks software in this paper.

\section{Structure Design}

\section{Working Principle of Soldering Machine.}

The structure sketch of soldering machine is shown in Fig. 1. The structure of soldering machine consists of three axis gantry, ball screw shaft, servo motor, solder gun, $\mathrm{X}$ axis module, $\mathrm{Y}$ axis module, $\mathrm{Z}$ axis module, angle encoder etc. The data is send to lower computer to control three axis motions by computer serial port, the process of automatic soldering is realized. The $\mathrm{X}$ and $\mathrm{Y}$ axis module is controlled by lower computer to ensure solder gun arrived at the solder joint and solder gun angle encoder adapt to the soldering angle. Solder wire is changed molten solder by the feed wire mechanism contact with solder gun, meanwhile the soldering process is realized by lower computer control $\mathrm{Z}$ axis module. 


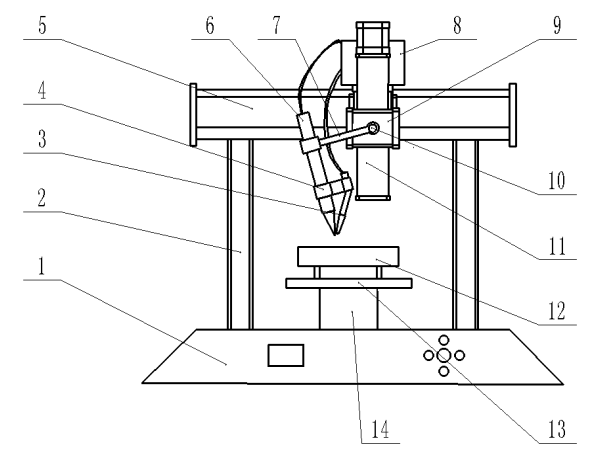

Fig. 1 The structure sketch of soldering machine

1- base, 2- frame, 3- solder wire conduit, 4- connecting rod, 5- X axis module, 6- solder gun, 7solder gun clamp, 8- feed wire mechanism, 9- connecting block, 10- angle encoder, 11- Z axis module, 12- PCB fixture, 13- workbench, 14- Y axis module

\section{Structure Simulation of Soldering Machine.}

At the present time, the engineering problems can be analyzed and simulated by a variety of software. SolidWorks 3D CAD solutions enable us to quickly transform new ideas into great products ${ }^{[5]}$. Hence, SolidWorks is used for structure analysis and design of solder machine.

Through preliminary determine of the key dimensions and assemble. SolidWorks is used for simulate the mechanical structure and check whether the structure is reasonable and motion interference. It is advantageous to the design parameters are further modified as well the optimization and design of mechanism is promoted.

In order to the aim of structure simple is achieved, the aluminum profile of standard parts $40 * 40$ * $500 \mathrm{~mm}$ is used for design the framework of soldering machine, and the ball screw shaft is fixed on the aluminum profile. To ensure the strength of aluminum profile, the strength of aluminum profile is analyzed by SolidWorks. Analysis result as shown in Fig. 2.

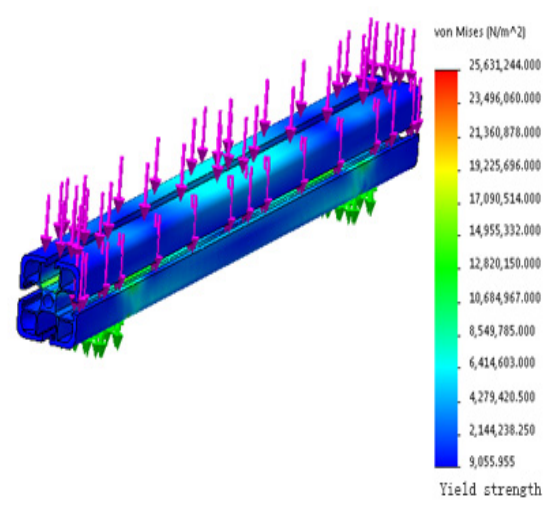

Fig. 2 Yield strength of aluminum profile

From the yield strength graph, it can be seen that the aluminum profile has enough strength as the framework of soldering machine.

\section{The Design of Control System}

\section{Control System of Lower Computer.}

According to the actual application of soldering machine, this control system should meet the following requirements:

(1) The function of the system control is performed by manual operation.

(2) The soldering angle is adjusted according to different situations by solder gun.

(3) The real-time feedback of solder status is obtained to the host computer software interface by lower computer.

According to the above requirements, the PIC-controlling is proposed in this paper. The PIC is a microcomputer that comprises a processor core, memory, and programmable input/output peripherals ${ }^{[6]}$. It can be installed a micro $C$ programming code, which is an important part of 
hardware control system. By the host computer recognition PCB file and encode data is sent to lower computer. PIC-controlling received the code and Y, X and Z axis module is controlled by servo motor, the soldering process is achieved.

Control flowchart of soldering machine system as shown in Fig. 3:

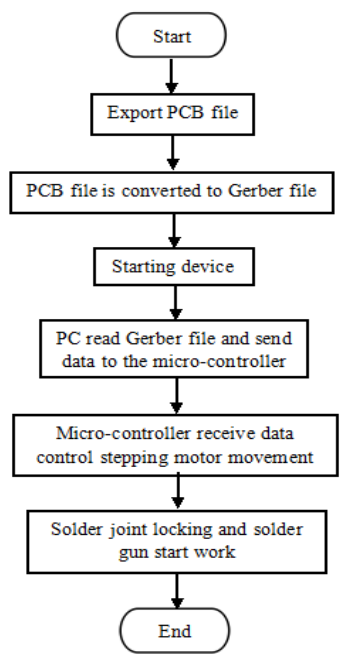

Fig. 3 Flow chart of control system

The control circuit of lower computer is comprised of central control unit, soldering temperature control circuit and motor drive circuit etc. The structure of the control system is shown in Fig. 4.

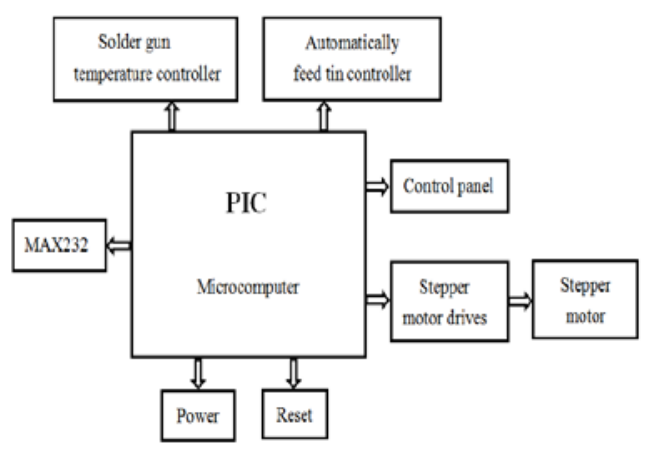

Fig. 4 Lower control system diagram

\section{Control System of Host Computer.}

The VC6.0 is adopted as the host computer software, the following functions are completed:

(1) PCB file is read for pad coordinate and solder gun path.

(2) The feedback is received and displayed on PC interface.

(3) The running status of lower computer is monitored.

PC software interface as shown in Fig. 5.

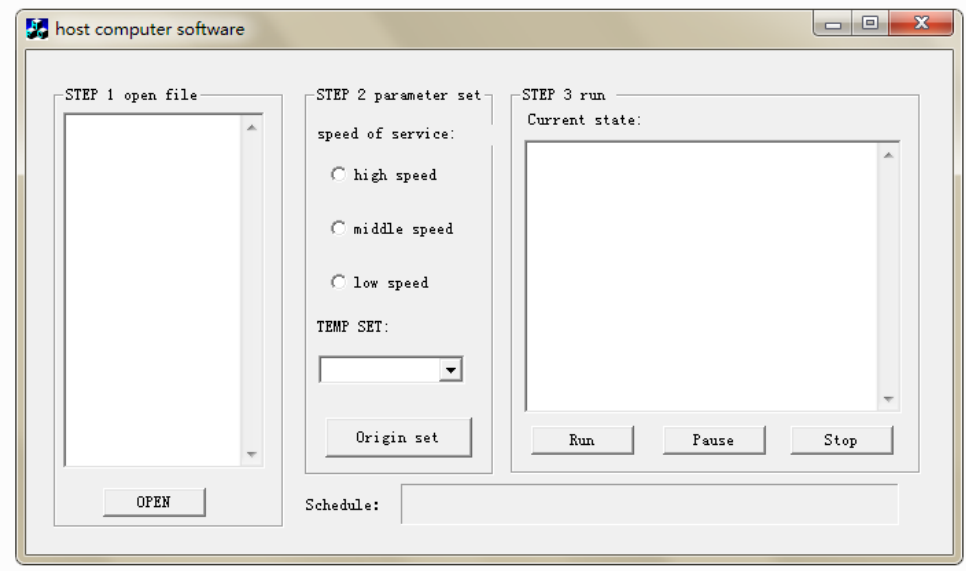

Fig. 5 Host computer software interface 


\section{Experimental Results}

The prototype of soldering machine is assembled based on hardware design as shown in Fig. 6, the soldering precision requirements are satisfied by the control system.

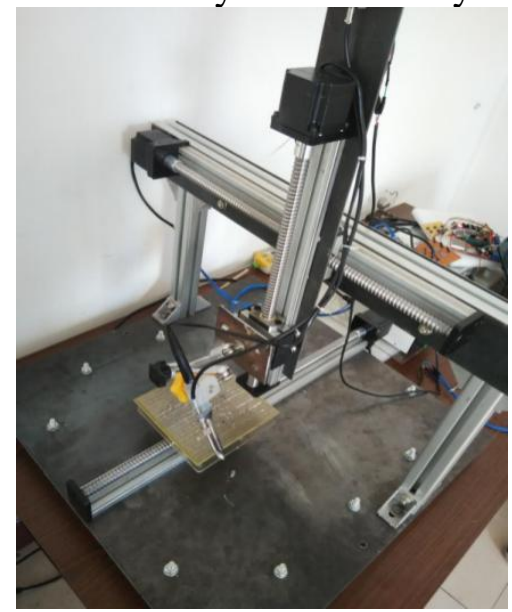

Fig. 6 The prototype of soldering machine

\section{Summary}

The soldering machine based on PIC-controlling is proposed in this paper, it has the advantages of low price, high accuracy, mature technology, which provide a good idea for traditional soldering machine, so that the soldering machine can be generalized and applied.

\section{Acknowledgments}

This work has been supported by the Innovation and Entrepreneurship Fund of Jiamusi University (xzyf2013-02) and the innovation and entrepreneurship students training program of Heilongjiang Province College Students (201410222018).

\section{References}

[1]. Mohd Sharizal Abdul Aziz, Mohd Zulkifly Abdullah, and Chu Yee Khor. Effects of Solder Temperature on Pin Through-Hole during Wave Soldering. Thermal-Fluid Structure Interaction Analysis. The Scientific World Journal. Vol 2014. Article ID 482363, 13 pages 2014.

[2]. M. S. Abdul Aziz, M.Z. Abdullah, and C. Y. Khor. Influence of PTH offset angel in wave soldering with thermal-coupling method. Soldering \& Surface Mount Technology. Vol. 26 (2014) No. 3, p. 97-109.

[3]. H-Q. Yang, S. Mazumder, S. Lowry, A. Krishnan, A. Przekwas, and L. Nguyen. Time-accurate, 3-D computation of wire sweep during plastic encapsulation of electronic components. Pressure Vessel Technol. Vol. 123 (2001) No. 4, p. 501-509.

[4]. http://usa.autodesk.com/asks/servlet/item?siteID=123112\&id=17628630.

[5]. http://www.gxsc.com/products/solidworks/overview.html.

[6]. Anuj Parikh, DhvaniShah, KrupaPoPat, Prof, Harish Narula. Blind Man Stick Using Programmable Interrupt Controller. Procedia Computer Science. Vol (2015) No. 48, p. 558-563. 